\title{
Polarimetric techniques for determining morphology and optical features of High Refractive Index dielectric nanoparticles size
}

\author{
Ángela I. Barreda, Juan M. Sanz, Rodrigo Alcaraz de la Osa, José M. Saiz, Fernando Moreno, \\ Francisco González*
}

\author{
Grupo de Óptica, Departamento de Física Aplicada, Universidad de Cantabria, Facultad de Ciencias, \\ Avda. Los Castros s/n, 39005. Santander. Spain
}

\begin{abstract}
The spectral evolution of the degree of linear polarization $\left(\mathrm{P}_{\mathrm{L}}\right)$ at a scattering angle of $90^{\circ}$ is studied numerically for high refractive index (HRI) dielectric spherical nanoparticles. The behaviour of $\mathrm{P}_{\mathrm{L}}\left(90^{\circ}\right)$ is analyzed as a function of the refractive index of the surrounding medium and the particle radius. We focus on the spectral region where both electric and magnetic resonances of order not higher than two are located for various semiconductor materials with low absorption. The spectral behavior of $\mathrm{P}_{\mathrm{L}}\left(90^{\circ}\right)$ has only a small, linear dependence on nanoparticle size R. This weak dependence makes it experimentally feasible to perform real-time retrievals of both the refractive index of the external medium and the NP size R. From an industrial point of view, pure materials are nonrealistic, since they can only be provided under certain conditions. For this reason, we also study the effect of contaminants on the resonances of silicon NPs by considering the spectral evolution of $\mathrm{P}_{\mathrm{L}}\left(90^{\circ}\right)$.
\end{abstract}

Keywords: Nanoparticles, polarization, sensing, sizing, contaminant, resonances.

\section{INTRODUCTION}

Nanotechnology has revolutionized science with important theoretical and practical developments. Particularly, the interaction of light with metallic nanoparticles (NPs) has been a very active field that has impacted many different areas. For instance, the development of new sensing techniques especially has attracted the attention of researchers in science and engineering ${ }^{1}$. When incident light illuminates a metallic NP, electron plasma oscillations are produced and, consequently, localized surface plasmons (LSPs) are generated. These coherent oscillations of the electron plasma depend on the material properties, the particle size and shape, and also on the wavelength of the incoming radiation and result in particular surface charge distributions ${ }^{2}$. At specific frequencies, resonances can be observed and strong enhancements of the electric field in the surroundings of the NPs may occur. Although most of the plasmonic studies of metallic NPs take advantage of the significant response of the plasma in the VIS-NIR range, their metallic nature is also the cause of their main disadvantage, ohmic losses.

High refractive index (HRI) dielectric NPs have been proposed as a solution for this problem because light can interact with these materials with negligible absorption ${ }^{3}$. In fact, instead of electronic plasma oscillations, certain distributions of displacement currents leading to whispering gallery-like modes are responsible for resonances in dielectric $\mathrm{NPs}^{4}$. These are located in well-defined spectral ranges, depend on the NPs size and shape, and can be of either electric or magnetic nature, although the particle magnetic permeability $\mu$ has a value of $1^{5}$. This magneto-dielectric behaviour has been vastly explored for some elements, such as Silicon and Germanium ${ }^{6-8}$, while the study of other semiconductor compounds has begun only recently ${ }^{9,10}$. There has been much work trying to exploit the experimental capabilities of HRI dielectric NPs in the last years ${ }^{11-14}$.

The resonances depend on the size, shape and optical properties of the NPs. For a given material, resonances are redshifted as NP size increases, and they also are influenced by the refractive index of the surrounding medium, $\mathrm{m}_{\text {med }}$. Particularly, for HRI dielectric materials, resonances are red-shifted as $\mathrm{m}_{\text {med }}$ increases, in addition to being strengthened $^{10}$. In previous works ${ }^{9,15,16}$, it was demonstrated that the degree of linear polarization of scattered light in a right-angle scattering configuration, $\mathrm{P}_{\mathrm{L}}\left(90^{\circ}\right)$, is a suitable parameter for extracting specific scattering properties of a system with a high degree of accuracy. It also has been shown to be a suitable parameter for determining particle size, also constituting a good sensor for studying multiple scattering problems of metallic systems ${ }^{16}$. For dielectric particles

Photonic and Phononic Properties of Engineered Nanostructures VI, edited by Ali Adibi, Shawn-Yu Lin, Axel Scherer, Proc. of SPIE Vol. 9756, 975627 • @ 2016 SPIE · CCC code: 0277-786X/16/\$18 · doi: 10.1117/12.2229488 
and, in particular, for those with high values of electric permittivity, the characteristics of $\mathrm{P}_{\mathrm{L}}\left(90^{\circ}\right)$ allow one to distinguish between their intrinsic electric or magnetic responses ${ }^{9,15}$. Another important factor that influences the behavior of the resonances is the purity of the NP material. In recent research, the effect of the purity of a silicon $(\mathrm{Si}) \mathrm{NP}$ on the light-scattering extinction spectrum was analyzed ${ }^{17}$. It was found that the magnetic resonances are more sensitive to changes in the purity than the electric resonances, and as the purity decreases, the magnetic resonances weakened. This behavior can be explained as the ohmic losses are much greater for the magnetic resonances than those for the electric resonances.

In this research, the measurement of $\mathrm{P}_{\mathrm{L}}\left(90^{\circ}\right)$ of the scattered light by isolated spherical NPs of representative HRI materials is analyzed as a function of the refractive index of its surrounding medium $\left(\mathrm{m}_{\text {med }} \in[1,2]\right)$ and the NP size $(R \in$ $[100,250] \mathrm{nm})$. As we will show, it allows both dependencies to be quantified simultaneously because their effects on $\mathrm{P}_{\mathrm{L}}\left(90^{\circ}\right)$ are concomitant but independent. Although the primary focus of this study is on Silicon ( $\left.\mathrm{Si}\right) \mathrm{NPs}$, we also consider the performance of four other HRI dielectric materials: Germanium (Ge), Aluminum Arsenide (AlAs), Aluminum Antimonide (AlSb) and Gallium Phosphide $(\mathrm{GaP})^{18}$. The most important common feature of these HRI materials is their low absorption in the spectral range analyzed (VIS-NIR) that can be considered insignificant in most cases. We also study the dependence of $\mathrm{P}_{\mathrm{L}}\left(90^{\circ}\right)$ on the purity of spherical Si NPs by examining their resonances.

This work is organized as follows: In Section 2 we will introduce the theoretical background. In Section 3, we will present the main results of this research, specifically, $\mathrm{P}_{\mathrm{L}}\left(90^{\circ}\right)$ as a useful parameter for sensing purposes. In addition, the capabilities of $\mathrm{P}_{\mathrm{L}}\left(90^{\circ}\right)$ in sizing NPs and in monitoring the NP purity will be discussed from a realistic point of view. Finally, results will be summarized in Section 4, where our concluding remarks will be presented.

\section{THEORETICAL METHODS}

Following the Lorenz-Mie formalism for scattering and absorption of light by small particles, the extinction and scattering cross- sections by a spherical particle are given by ${ }^{19}$ :

$$
\begin{aligned}
& C_{\text {ext }}=\frac{2 \cdot \pi}{k^{2}} \sum_{n=1}^{\infty}(2 \cdot n+1) \operatorname{Re}\left\{a_{n}+b_{n}\right\} \\
& C_{\text {sca }}=\frac{2 \cdot \pi}{k^{2}} \sum_{n=1}^{\infty}(2 \cdot n+1)\left(\left|a_{n}\right|^{2}+\left|b_{n}\right|^{2}\right)
\end{aligned}
$$

where $a_{n}$ and $b_{n}$ are the well-known scattering coefficients of the Lorenz-Mie theory. These depend on the particle size and on the electric and magnetic properties of the NP relative to its surrounding medium. The wavenumber $k$ is defined as $\left(2 \pi \mathrm{m}_{\mathrm{med}}\right) / \lambda$, where $\lambda$ is the wavelength of incident light in the surrounding medium. Physically, coefficients $a_{n}$ and $b_{n}$ represent the weighting factors of the different multipolar contributions of order $n$, electric or magnetic respectively. In particular, $a_{1}$ and $b_{1}$ correspond to the electric and magnetic dipolar modes, respectively, and $a_{2}$ and $b_{2}$ correspond to the quadrupolar modes, respectively. The efficiencies are related to the cross-sections through the following expression: $\mathrm{Q}=$ $\mathrm{C} / \mathrm{G}$, being $\mathrm{G}$ the particle cross-sectional area projected onto a plane perpendicular to the incident beam.

In the same way, the intensities of light scattered by NPs are related to $a_{n}$ and $b_{n}$ through the scattering matrix elements, which depend on the scattering direction. For linearly polarized light, the components parallel $\left(\mathrm{E}_{\mathrm{pSca}}\right)$ and perpendicular $\left(\mathrm{E}_{\mathrm{sSca}}\right)$ to the scattering plane are related to the incident fields, $\mathrm{E}_{\mathrm{pInc}}$ and $\mathrm{I}_{\text {sInc }}$ by a diagonal scattering matrix:

$$
\left(\begin{array}{c}
E_{p S c a} \\
E_{s S c a}
\end{array}\right)=\frac{e^{i \cdot k \cdot(r-z)}}{-i \cdot k \cdot r}\left(\begin{array}{cc}
S_{2} & 0 \\
0 & S_{1}
\end{array}\right)\left(\begin{array}{l}
E_{p I n c} \\
E_{s I n c}
\end{array}\right)
$$


where $S_{1}$ and $S_{2}$ are the diagonal elements of the scattering matrix, which are defined from the Lorenz-Mie scattering coefficients $a_{n}$ and $b_{n}$ and the angle-dependent functions $\pi_{\mathrm{n}}$ and $\tau_{\mathrm{n}}$ as ${ }^{19}$

$$
\begin{aligned}
& S_{1}=\sum_{n} \frac{2 \cdot n+1}{n \cdot(n+1)}\left(a_{n} \cdot \pi_{n}+b_{n} \cdot \tau_{n}\right) \\
& S_{2}=\sum_{n} \frac{2 \cdot n+1}{n \cdot(n+1)}\left(a_{n} \cdot \tau_{n}+b_{n} \cdot \pi_{n}\right)
\end{aligned}
$$

The total scattered intensities with polarization parallel, $\mathrm{I}_{\mathrm{psca}}$, and perpendicular, $\mathrm{I}_{\mathrm{sSca}}$, to the scattering plane are proportional to $\left|S_{2}\right|^{2}$ and $\left|S_{1}\right|^{2}$, respectively, and the linear polarization degree of the scattered light in a given direction $\theta$ can be defined as:

$$
P_{L}(\theta)=\frac{I_{s S c a}-I_{p S c a}}{I_{s S c a}+I_{p S c a}}
$$

In particular, at right-angle scattering configuration $\left(\theta=90^{\circ}\right)$, and for a NP size smaller than the incident wavelength, we retain only the first two orders in Eq. (3) ${ }^{9}$ :

$$
P_{L}\left(90^{\mathrm{o}}\right)=\frac{9\left|a_{1}\right|^{2}+25\left|\mathrm{~b}_{2}\right|^{2}+30 \operatorname{Re}\left\{\mathrm{b}_{1} a_{2} *\right\}-9\left|\mathrm{~b}_{1}\right|^{2}-25\left|\mathrm{a}_{2}\right|^{2}-30 \operatorname{Re}\left\{a_{1} b_{2} *\right\}}{9\left|a_{1}\right|^{2}+25\left|\mathrm{~b}_{2}\right|^{2}+30 \operatorname{Re}\left\{\mathrm{b}_{1} a_{2} *\right\}+9\left|\mathrm{~b}_{1}\right|^{2}+25\left|\mathrm{a}_{2}\right|^{2}+30 \operatorname{Re}\left\{a_{1} b_{2} *\right\}}
$$

Indirectly, Eq. (5) may provide information about both the NP size and its surrounding medium based on the value of the polarimetric observation $\mathrm{P}_{\mathrm{L}}\left(90^{\circ}\right)$. This is because the scattering coefficients are related to the particle size and the relative refractive index. From an experimental point of view, right-angle-detection measurements are simple to execute and were used recently to analyze the spectral behaviour of $\mathrm{P}_{\mathrm{L}}\left(90^{\circ}\right)$ for a single particle made of an HRI material ${ }^{9}$. Although in experimental measurements the scattered light is collected and the signal is integrated over the solid angle of detection, in this work it was shown that $\mathrm{P}_{\mathrm{L}}\left(90^{\circ}\right)$ could be measured accurately in an experimentally feasible way. Figure 1 shows a set-up of the scattering geometry to perform right-angle-detection measurements.

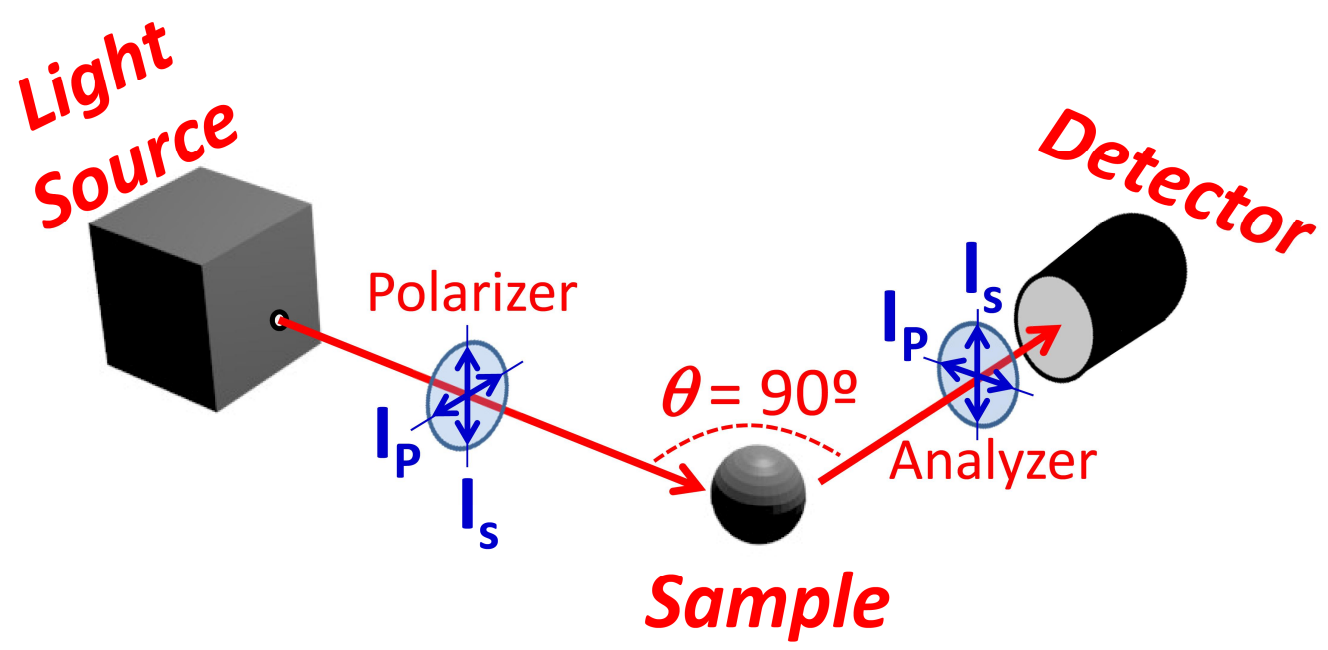

Figure 1. Experimental setup for carrying out the measurements of $\mathrm{P}_{\mathrm{L}}\left(90^{\circ}\right)$. 


\section{RESULTS}

\section{1 $\mathbf{P}_{\mathrm{L}}\left(\mathbf{9 0}^{\circ}\right)$ in sensing}

We have analyzed the spectral values of $\mathrm{P}_{\mathrm{L}}\left(90^{\circ}\right)$ for a spherical Si NP of radius $(\mathrm{R}=200 \mathrm{~nm})$ as a function of $\mathrm{m}_{\text {med }}$. Values of all resonances evolve with $\mathrm{m}_{\text {med }}$, so that an estimate of $\mathrm{m}_{\text {med }}$ can be obtained through the values of $\mathrm{P}_{\mathrm{L}}\left(90^{\circ}\right)$, for those wavelengths where resonances occur, $\lambda_{\text {res. }}$. The resonances spectral position $\lambda_{\text {res }}$ red-shifts as $m_{\text {med }}$ increases for both $a_{1}$ and $b_{2}$, whereas both the red-shift and the minimum in $b_{1}$ are hardly visible for $m_{\text {med }}>1.5$. The red-shift is more significant for the electric dipolar resonance $a_{1}$ than for the magnetic quadrupolar resonance $b_{2}$.

In order to evaluate the accuracy of $\mathrm{P}_{\mathrm{L}}\left(90^{\circ}\right)$ as an estimator of $\mathrm{m}_{\text {med }}$, it is necessary to analyze the positions of the resonances as a function of the wavelength at which they occur $\lambda_{\text {res }}$, i.e., $\frac{\partial P_{L}\left(90^{\circ}\right)}{\partial \lambda}=0$

The sensitivity of $\mathrm{P}_{\mathrm{L}}\left(90^{\circ}\right)$ to $\mathrm{m}_{\text {med }}$ can be obtained through the parameter $\mathrm{S}_{\mathrm{m}}\left(90^{\circ}\right)$, defined as

$$
S_{m}\left(90^{\circ}\right)=\left|\left(\frac{\partial P_{L}\left(90^{\circ}\right)}{\partial m_{\text {med }}}\right)_{\lambda_{\text {res }}}\right|
$$

The value of $\mathrm{S}_{\mathrm{m}}\left(90^{\circ}\right)$ shows the relative change in $\mathrm{P}_{\mathrm{L}}\left(90^{\circ}\right)$ as a function of the refractive index of the surrounding medium. This parameter is measured in inverse Refractive Index Units $\left(\mathrm{RIU}^{-1}\right)^{20}$ and can be used to evaluate how the values of $\mathrm{P}_{\mathrm{L}}\left(90^{\circ}\right)$ in the resonances are influenced by $\mathrm{m}_{\mathrm{med}}$ variations. Accordingly, the greater the value of $\mathrm{S}_{\mathrm{m}}\left(90^{\circ}\right)$, the more accurate the measurement of $\mathrm{m}_{\text {med }}$. The analysis of the $\mathrm{P}_{\mathrm{L}}\left(90^{\circ}\right)$ values with respect to $\mathrm{m}_{\text {med }}$ has been analyzed for a Si R $=200 \mathrm{~nm} \mathrm{NP}$ and at those wavelengths where $\mathrm{a}_{1}, \mathrm{~b}_{1}$ and $\mathrm{b}_{2}$ resonances take place $\left(\lambda_{\text {res }}\right)$. On this basis, we have evaluated $\mathrm{S}_{\mathrm{m}}\left(90^{\circ}\right)$ averaged over a given $\mathrm{m}_{\text {med }}$ range. Table 1 provides an example of how this parameter can be used to quantify the resonance behaviour of different materials. It shows $\mathrm{S}_{\mathrm{m}}\left(90^{\circ}\right)$ values for all the considered semiconductors: Silicon, Germanium, Aluminum Arsenide, Aluminum Antimonide and Gallium Phosphide at $\lambda_{\text {res }}$ and averaged over the range $\mathrm{m}_{\mathrm{med}} \in[1,2]$.

Table 1. $\mathrm{P}_{\mathrm{L}}\left(90^{\circ}\right)$ sensitivity $\left(\mathrm{S}_{\mathrm{m}}\left(90^{\circ}\right)\right)$ to $\mathrm{m}_{\text {med }}\left(\mathrm{m}_{\mathrm{med}} \in[1,2]\right)$ at resonances for the selected materials (particle radius $\mathrm{R}=200$ $\mathrm{nm})$.

\begin{tabular}{|l|l|l|l|}
\hline \multicolumn{5}{|c|}{$\mathbf{S}_{\mathbf{m}}\left(\mathbf{9 0}^{\circ}\right)$} \\
\hline $\begin{array}{l}\text { Semiconductor } \\
\text { Material }\end{array}$ & Quadrupolar Magnetic & Dipolar Electric & Dipolar Magnetic \\
\hline Germanium & 0 & 0.99 & 0.81 \\
\hline Gallium Phosphide & 1.73 & 1.37 & 0.63 \\
\hline Aluminum Arsenide & 1.67 & 1.23 & 0.60 \\
\hline Aluminum Antimonide & 1.70 & 1.35 & 0.71 \\
\hline Silicon & 1.58 & 1.40 & 0.78 \\
\hline
\end{tabular}

The sensitivity values of $\mathrm{P}_{\mathrm{L}}\left(90^{\circ}\right)$ for all the materials and resonances mentioned thus far are large enough to be used to make experimental estimates of $\mathrm{m}_{\text {med. }}$. As shown in Table 1 , most of the $\mathrm{S}_{\mathrm{m}}\left(90^{\circ}\right)$ values are larger than $0.5 \mathrm{RIU} \mathrm{U}^{-1}$ for both 
electric and magnetic dipolar resonances, and increase toward $1.5 \mathrm{RIU}^{-1}$ for the quadrupolar magnetic resonance. Obviously, $\mathrm{S}_{\mathrm{m}}\left(90^{\circ}\right)$ values on the order of $1.0 \mathrm{RIU}^{-1}$ imply similar changes in both $\mathrm{P}_{\mathrm{L}}\left(90^{\circ}\right)$ and in $\mathrm{m}_{\text {med. }}$. From a realistic point of view, the error margin of most polarimetric arrangements is smaller than $1 \%$ at present ${ }^{21}$, so that $\mathrm{S}_{\mathrm{m}}\left(90^{\circ}\right) \sim 1.0$ $\mathrm{RIU}^{-1}$ suggests an accuracy in sensing around $0.01 \mathrm{RIU}$ at worst.

\section{2 $\mathbf{P}_{\mathrm{L}}\left(90^{\circ}\right)$ in sizing}

As the NP size increases, the resonance is red-shifted toward larger wavelengths. In Table 2 we show the spectral positions of the quadrupolar magnetic, dipolar electric and dipolar magnetic resonances as a function of the NP size for a Si spherical NP embedded in vacuum, $\mathrm{m}_{\text {med }}=1.0$. The raddi $\mathrm{R}$ range from 100 to $250 \mathrm{~nm}$.

Table 2. Spectral location of the local maximum or minimum in $\mathrm{P}_{\mathrm{L}}\left(90^{\circ}\right)$ for a $\mathrm{Si}$ spherical $\mathrm{NP}\left(\mathrm{m}_{\mathrm{med}}=1.0\right)$ as a function of the particle size $(\mathrm{R})$.

\begin{tabular}{|l|l|l|l|}
\hline Radius (nm) & Quadrupolar magnetic & Dipolar electric & Dipolar magnetic \\
\hline 100 & $\begin{array}{l}\text { No data in the analyzed spectral } \\
\text { region }\end{array}$ & 606 & 775 \\
\hline 125 & 686 & 725 & 937 \\
\hline 150 & 799 & 840 & 1113 \\
\hline 175 & 909 & 993 & 1290 \\
\hline 200 & 1034 & 1118 & 1458 \\
\hline 225 & 1152 & 1256 & 1635 \\
\hline 250 & 1278 & 1375 & 1811 \\
\hline
\end{tabular}

In Table 3 we show the values of the resonances in the $\mathrm{P}_{\mathrm{L}}\left(90^{\circ}\right)$ spectra for the same considered NP sizes as previously. From these data and using Eq. (7) we can obtain the sensitivity of this parameter to changes in the NP size. The sensitivity of $\mathrm{P}_{\mathrm{L}}\left(90^{\circ}\right)$ on $\mathrm{R}$ is measured in $\mathrm{nm}^{-1}$ and it demonstrates that $\mathrm{P}_{\mathrm{L}}\left(90^{\circ}\right)$ is relatively insensitive to the size of the NP. Typical values are less than $0.001 \mathrm{~nm}^{-1}$.

$$
S_{R}\left(90^{\circ}\right)=\left|\left(\frac{\partial P_{L}\left(90^{\circ}\right)}{\partial R}\right)_{\lambda_{\text {res }}}\right|
$$


Table 3. Values of the local maximum or minimum in $\mathrm{P}_{\mathrm{L}}\left(90^{\circ}\right)$ for a Si spherical $\mathrm{NP}\left(\mathrm{m}_{\mathrm{med}}=1.0\right)$ as a function of the particle size (R).

\begin{tabular}{|l|l|l|l|}
\hline Radius (nm) & Quadrupolar magnetic & Dipolar electric & Dipolar magnetic \\
\hline 100 & $\begin{array}{l}\text { No data in the analyzed } \\
\text { spectral region }\end{array}$ & 0.75 & -0.72 \\
\hline 125 & 0.72 & 0.71 & -0.69 \\
\hline 150 & 0.76 & 0.69 & -0.69 \\
\hline 175 & 0.78 & 0.67 & -0.68 \\
\hline 200 & 0.79 & 0.67 & -0.67 \\
\hline 225 & 0.79 & 0.64 & -0.67 \\
\hline 250 & 0.79 & 0.64 & -0.66 \\
\hline
\end{tabular}

The magnitude of $\mathrm{P}_{\mathrm{L}}\left(90^{\circ}\right)$, being almost independent of particle size, has high potential as a sensing parameter, especially NPs submerged in liquids. Since the magnitude of $\mathrm{P}_{\mathrm{L}}\left(90^{\circ}\right)$ only has a strong dependence on $\mathrm{m}_{\text {med }}$, it can be used for characterizing the external medium. With $\mathrm{m}_{\text {med }}$ now a known quantity, the spectral position of the resonance can be used to determine NP size $\mathrm{R}$

Determining NP size R is a common characterization problem. Suspensions of NPs in a liquid medium are typically polydisperse, with size deviations ranging from 10 to 0.1 times a mean value $\mathrm{R}$. The ability to characterize both the particle size $R$ and to estimate the medium refractive index $m_{\text {med }}$ simultaneously is what makes $P_{L}\left(90^{\circ}\right)$ a promising experimental tool.

\section{3 $\mathbf{P}_{\mathrm{L}}\left(\mathbf{9 0}^{\circ}\right)$ for determining purity grade of NPs}

From an industrial point of view, pure materials are nonrealistic, since they can only be provided under certain conditions. For this reason, in this section, we analyze the effect of NP impurity on the linear polarization degree $\mathrm{P}_{\mathrm{L}}\left(90^{\circ}\right)$, and consider its use to determine the purity grade of the NP material. As the contamination increases and NP purity decreases, absorption increases. The resonances weaken. This effect is more noticeable for the magnetic resonances than for the electric ones, as it was mentioned in the introduction.

We have obtained the value of the linear polarization $\mathrm{P}_{\mathrm{L}}\left(90^{\circ}\right)$ at the wavelengths where the resonances appear, $\lambda_{\text {res }}$, for different concentrations, $100 \%, 99.88 \%, 99.73 \%, 99.55 \%, 99.37 \%, 99.16 \%$ and $98.98 \%$. The sensitivity of $\mathrm{P}_{\mathrm{L}}\left(90^{\circ}\right)$ on the purity is obtained through the following parameter: $\mathrm{S}_{\mathrm{P}}\left(90^{\circ}\right)$.

$$
S_{P}\left(90^{\circ}\right)=\left|\left(\frac{\partial P_{L}\left(90^{\circ}\right)}{\partial P \operatorname{urity}(\%)}\right)_{\lambda_{\text {res }}}\right|
$$

In Table 4 . We show the sensitivity of linear polarization degree $\mathrm{S}_{\mathrm{P}}\left(90^{\circ}\right)$ to NP purity where the percent Si ranges from $100 \%$ to $98.98 \%$ for a $\mathrm{R}=200 \mathrm{~nm}$ spherical $\mathrm{NP}$ embedded in vacuum. 
Table 4. Sensitivity of linear polarization degree $\mathrm{S}_{\mathrm{P}}\left(90^{\circ}\right)$ to NP purity where the percent Si ranges from $100 \%$ to $98.98 \%$ for a $\mathrm{R}=$ $200 \mathrm{~nm}$ spherical NP embedded in vacuum.

\begin{tabular}{|l|l|l|}
\hline \multicolumn{2}{|c|}{$\mathrm{S}_{\mathbf{P}}\left(90^{\circ}\right)$} \\
\hline Quadrupolar magnetic & Dipolar electric & Dipolar magnetic \\
\hline 0.56 & 0.081 & 0.27 \\
\hline
\end{tabular}

From the Table 4. it is clear that the magnetic resonances are most sensitive to changes in the NP purity than the electric ones, specially the quadrupolar magnetic resonance.

\section{CONCLUSION}

In this manuscript, we have discussed the use of the linear polarization degree of light scattered by HRI dielectric NPs at right-angle configuration, $\mathrm{P}_{\mathrm{L}}\left(90^{\circ}\right)$, as a tool to characterize NPs and the medium they are suspended within. Lorenz-Mie calculations of spherical NPs made of $\mathrm{Si}, \mathrm{Ge}, \mathrm{AlAs}, \mathrm{AlSb}$ and $\mathrm{GaP}$ were made at several radii $(\mathrm{R} \in[100,250] \mathrm{nm})$ and refractive indices of the surrounding medium $\left(\mathrm{m}_{\text {med }} \in[1.0,2.0]\right)$. The obtained $\mathrm{P}_{\mathrm{L}}\left(90^{\circ}\right)$ values for the analyzed resonances (quadrupolar magnetic, electric and magnetic dipolar) have only a small, nearly linear dependence on the size of the NP (R), and a much larger dependence on $\mathrm{m}_{\text {med. }}$. We have also demonstrated that $\mathrm{P}_{\mathrm{L}}\left(90^{\circ}\right)$ is a useful tool for determining the purity grade of NPs. We have observed that an increase in NP contamination affects the dipolar and quadrupolar magnetic resonances more than the electric resonances, with the quadrupolar magnetic resonance being the most sensitive one.

\section{ACKNOWLEDGMENTS}

This research was supported by MICINN (Spanish Ministry of Science and Innovation, project FIS2013-45854-P). Ángela I. Barreda want to express their gratitude to the University of Cantabria for her FPU grant.

\section{REFERENCES}

[1] Prasad, P. N., [Nanophotonics], John Wiley \& Sons, Inc., (2004).

[2] Kelly, K. L., Coronado, E., Zhao, L. L., and Schatz, G., "The optical properties of metal nanoparticles: the influence of size, shape and dielectric environment," J. Phys. Chem. B 107, 668-677 (2003).

[3] Forouhi, A. and Bloomer, I., "Optical properties of crystalline semiconductors and dielectrics," Phys. Rev. B $38,1865-1874$ (1988).

[4] García-Etxarri, A., Gómez-Medina, R., Froufe-Perez, L. S., López, C., Chantada, L., Scheffold, F., Aizpurua, J., Nieto-Vesperinas, M., and Sáenz, J. J., "Strong magnetic response of submicron silicon particles in the infrared," Opt. Express 19, 4815-4826 (2011).

[5] Fu, Y. H., Kuznetsov A. I., Miroshnichenko A. E., Yu, Y. F., and Luk'yanchuk B., "Directional visible light scattering by silicon nanoparticles," Nat. Commun. 4, 1527 (2013).

[6] Shi, L., Harris, J. T., Fenollosa, R., Rodriguez, I., Lu, X., Korgel, B. A., and Meseguer, F., "Monodisperse silicon nanocavities and photonic crystals with magnetic response in the optical region," Nat. Commun. 4, 1904 (2013).

[7] Evlyukhin, A. B., Reinhardt, C., Seidel, A., Luk'yanchuk, B. and Chichkov B. N., "Optical response features of si-nanoparticle arrays," Phys. Rev. B 82(4), 045404 (2010). 
[8] Gómez-Medina, R., García-Cámara, B., Suárez-Lacalle, I., González, F., Moreno, F., Nieto-Vesperinas, M., and Sáenz, J. J, "Electric and magnetic dipolar response of germanium nanospheres: interference effects, scattering anisotropy, and optical forces," J. Nanophoton. 5(1), 053512 (2011).

[9] Geffrin, J. M., García-Cámara, B., Gómez-Medina, R., Albella, P., Froufe-Pérez, L., Eyraud, C., Litman, A., Vaillon, R., González, F., Nieto-Vesperinas, M., Sáenz, J. J., and Moreno, F., "Magnetic and electric coherence in forward- and back-scattered electromagnetic waves by a single dielectric subwavelength sphere," Nat. Commun. 3, 1171 (2012).

[10] García-Cámara, B., Gómez-Medina, R., Sáenz, J. J., and Sepúlveda, B., "Sensing with magnetic dipolar resonances in semiconductor nanospheres," Opt. Express 21, 23007-23020 (2013).

[11] Evlyukhin, A. B., Novikov, S. M., Zywietz, U., Eriksen, R. L., Reinhardt, C., Bozhevolnyi, S. I., and Chichkov, B. N., "Demonstration of magnetic dipole resonances of dielectric nanospheres in the visible region," Nano Lett. 12, 3749-3755 (2012).

[12] Shi, L., Tuzer, T. U., Fenollosa, R., and Meseguer, F., "A new dielectric metamaterial building block with a strong magnetic response in the sub-1.5-micrometer region: silicon colloid nanocavities," Adv. Mater. 24(44), 5934-5938 (2012).

[13] Kuznetsov, A. I., Miroshnichenko, A. E., Fu, Y. H., Zhang, J., and Luk'yanchuk, B., “Magnetic light,” Sci. Rep. 2, 492 (2012).

[14]Zywietz, U., Evlyukhin, A. B., Reinhardt, C., and Chichkov, B. N., "Laser printing of silicon nanoparticles with resonant optical electric and magnetic responses," Nat. Commun. 5, 3402 (2014).

[15] García-Cámara, B., González, F., and Moreno, F., "Linear polarization degree for detecting magnetic properties of small particles," Opt. Lett. 35(23), 4084-4086 (2010).

[16] Setién, B., Albella, P., Saiz, J. M., González, F., and Moreno, F., "Spectral behavior of the linear polarization degree at right-angle scattering configuration for nanoparticle systems," New J. Phys. 12, 103031 (2010).

[17] Sanz, J. M., Alcaraz de la Osa, R., Barreda, A. I., Saiz, J. M., González, F., and Moreno, F., "Influence of pollutants in the magneto-dielectric response of silicon nanoparticles," Opt. Lett. 39(11), 3142-3144 (2014).

[18] Palik, E. D., [Handbook of Optical Constants of Solids], Academic Press, (1998).

[19] Bohren, C. F. and Huffman, D. R., [Absorption and Scattering of Light by Small Particles], John Wiley \& Sons, Inc., (1983).

[20]Xia, Y. and Hallas, N. J., "Shape-controlled synthesis and surface plasmonic properties of metallic nanostructures," MRS Bulletin 30, 338-348 (2005).

[21] Sanz, J. M., Saiz, J. M., González, F., and Moreno, F., "Polar decomposition of the mueller matrix: a polarimetric rule of thumb for square-profile surface structure recognition," Appl. Opt. 50, 3781-3788 (2011). 